\title{
Fatores de risco para lesão renal aguda em pacientes clínicos intensivos
}

\author{
Risk factors for acute renal injury in intensive clinical patients \\ Factores de riesgo para lesión renal aguda en pacientes clínicos intensivos
}

Cariston Rodrigo Benichel it hitps://orcid.org/0000-0003-2781-0292']

Silmara Meneguin io https://orcid.org/0000-0003-3853-5134

\section{Como citar:}

Benichel CR, Meneguin S. Fatores de risco para lesão renal aguda em pacientes clínicos intensivos. Acta Paul Enferm. 2020;33:e-APE20190064 acta-ape/2020A00064

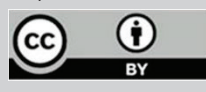

Descritores

Lesão renal aguda; Fatores de risco; Cuidados intensivos; Pacientes internados

Keywords

Acute kidney injury; Risk factors; Critical care

Inpatients;

Descriptores

Lesión renal aguda; Factores de riesgo; Cuidados críticos: Pacientes internos

Submetido 20 de Março de 2019

Aceito

14 de Agosto de 2019

Autor correspondente Cariston Rodrigo Benichel https://orcid.org/0000-0003-2781-0292 E-mail: c.benichel@hotmail.com

\section{Resumo}

Objetivo: Identificar prevalência e fatores associados à lesão renal aguda em pacientes clínicos intensivos, e compará-Ios com um grupo controle; analisar se a coexistência de fatores constitui preditor de risco para 0 desenvolvimento de lesão renal aguda.

Métodos: Estudo caso-controle, com abordagem quantitativa, realizado em unidade de terapia intensiva geral adulto do interior de São Paulo, Brasil, com 205 pacientes que desenvolveram lesão renal aguda e o mesmo número de controles, durante os anos de 2014 e 2015. Coleta de dados realizada mediante levantamento dos registros de prontuário. Relações foram estatisticamente significativas se $p<0,05$.

Resultados: A prevalência de lesão renal aguda foi de 7,5\% e os principais fatores associados foram: hipertensão arterial $(p=0,004 ; 0 R=1,9615 ; \quad I C=1,0491-3,6645)$; hipovolemia $(p=0,006 ; 0 R=5,6071$; $I C=1,6382-19,1854)$; insuficiência cardíaca $(p=0,003 ; 0 R=5,3123 ; \quad I C=1,7521-16,1051)$; noradrenalina $(p<0,0001 ; O R=9,4913 ; I C=4,4824-20,0981)$; dopamina $(p=0,0009 ; \quad O R=3,5212 ; I C=1,6701-7,4242)$; dobutamina $(p=0,0131 ; \quad O R=5,2612 ; \quad I C=1,4172-19,5323) ; \quad$ e antibióticos simultâneos $(p<0,0001$; $\mathrm{OR}=3,7881 ; \mathrm{IC}=2,0253-7,0884)$. A coexistência de mais de três fatores de risco foi estatisticamente significante para lesão renal aguda ( $\mathrm{p}<0,0001 ; 0 R=5,0074$; $I C=2,5601-9,7936)$.

Conclusão: A lesão renal aguda é um evento multifatorial que se associou à doença de base, às complicações decorrentes da gravidade dos participantes e à utilização de medicamentos nefrotóxicos. Ter três ou mais fatores de risco aumentou as chances para o desenvolvimento da doença.

\section{Abstract}

Objective: Identify the prevalence and associated factors of acute renal injury in intensive clinical patients and compare them with a control group; analyze if the coexistence of factors serves as a predictor for the risk of developing acute renal injury.

Method: Case-control study with a quantitative approach, developed at a general adult intensive care unity in the interior of São Paulo, Brazil, involving 205 patients who developed acute renal injury and the same number of controls, during 2014 and 2015. Data were collected through a survey of patient file records. Relationships were statistically significant if $p<0.05$.

Results: The prevalence of acute renal injury was $7.5 \%$ and the main associated factors were: arterial hypertension ( $\mathrm{p}=0.004 ; \mathrm{OR}=1.9615 ; \mathrm{Cl}=1.0491-3.6645)$; hypovolemia $(\mathrm{p}=0.006 ; \mathrm{R}=5.6071 ; \mathrm{Cl}=1.6382$ 19.1854); heart failure $(p=0.003 ; 0 R=5.3123 ; C l=1.7521-16.1051)$; noradrenaline $(p<0.0001 ; 0 R=9.4913$; $\mathrm{Cl}=4.4824-20.0981)$; dopamine $(\mathrm{p}=0.0009 ; \mathrm{OR}=3.5212 ; \mathrm{Cl}=1.6701-7.4242) ;$ dobutamine $(\mathrm{p}=0.0131$; $\mathrm{OR}=5.2612 ; \mathrm{Cl}=1.4172-19.5323)$; and simultaneous antibiotics $(\mathrm{p}<0.0001 ; 0 \mathrm{R}=3.7881 ; \mathrm{Cl}=2.0253$ - 
7.0884). The coexistence of more than three risk factors was statistically significant for acute renal injury ( $\mathrm{p}<0.0001 ; \mathrm{OR}=5.0074 ; \mathrm{Cl}=2.5601-9.7936)$. Conclusion: Acute renal injury is a multifactorial event associated with the baseline disease, the complications deriving from the severity of the patients' condition and the use of nephrotoxic drugs. Having three or more risk factors increased the chances for the development of the disease.

\section{Resumen}

Objetivo: Identificar prevalencia y factores asociados a la lesión renal aguda en pacientes clínicos intensivos y compararlos con un grupo de control; analizar si la coexistencia de factores constituye predictor de riesgo para el desarrollo de lesión renal aguda.

Métodos: Estudio caso-control, con enfoque cuantitativo, realizado en unidad de cuidados intensivos general adulto del interior del estado de São Paulo, Brasil, con 205 pacientes que desarrollaron lesión renal aguda y el mismo número de controles, durante los años 2014 y 2015 . Recolección de datos realizada mediante recopilación de registros de historia clínica. Relaciones fueron estadísticamente significativas si p<0,05.

Resultados: La prevalencia de lesión renal aguda fue de 7,5\% y los principales factores asociados fueron: hipertensión arterial ( $p=0,004 ; 0 R=1,9615$; $I C=1,0491-3,6645)$; hipovolemia $(p=0,006 ; 0 R=5,6071 ; \quad I C=1,6382-19,1854)$; insuficiencia cardíaca $(p=0,003 ; 0 R=5,3123 ; I C=1,7521-16,1051)$; noradrenalina $(p<0,0001 ; \quad O R=9,4913 ; \quad I C=4,4824-20,0981) ; \quad$ dopamina $(p=0,0009 ; \quad O R=3,5212 ; \quad I C=1,6701-7,4242) ; \quad$ dobutamina $(p=0,0131$; $\mathrm{OR}=5,2612 ; \mathrm{IC}=1,4172-19,5323) ; \mathrm{y}$ antibióticos simultáneos $(\mathrm{p}<0,0001 ; \mathrm{R}=3,7881 ; \mathrm{I}=2,0253-7,0884)$. La coexistencia de más de tres factores de riesgo fue estadísticamente significante para la lesión renal aguda ( $\mathrm{p}<0,0001 ; 0 \mathrm{R}=5,0074 ; \mathrm{IC}=2,5601-9,7936)$.

Conclusión: La lesión renal aguda es un evento multifactorial que se asoció a la enfermedad de base, a las complicaciones resultantes de la gravedad de los participantes y a la utilización de medicamentos nefrotóxicos. Tener tres o más factores de riesgo aumentó las chances de desarrollo de la enfermedad.

\section{Introdução}

A Lesão Renal Aguda (LRA) é uma doença sistêmica, multifatorial e que contribui para aumento $\mathrm{da}$ morbimortalidade de pacientes internados em Unidade de Terapia Intensiva (UTI). ${ }^{(1)}$ No ambiente hospitalar, é uma das complicaçôes mais frequentes em pacientes hospitalizados, cuja prevalência oscila entre 15 e $30 \%$, porém, nas UTI, este valor é praticamente duplicado. ${ }^{(2)}$ Estima-se que $13 \%$ dos pacientes destas unidades seráo tratados com terapia de substituição renal, destes, 50 a 60\% irão a óbito. $(2,3)$

Dentre os fatores de risco para desenvolver LRA, destacam-se as doenças clínicas preexistentes, intervenções terapêuticas, além da susceptibilidade individual que podem repercutir na função renal. Também há relação com o processo de envelhecimento, atrelado às doenças crônico-degenerativas e alterações renais morfofuncionais. ${ }^{(4)}$

O diagnóstico precoce da LRA está diretamente relacionado ao melhor prognóstico de pacientes clínicos críticos, e dentre as estratégias comumente utilizadas, tem-se a mensuração de marcadores biológicos, a partir da análise de dados laboratoriais, que sinalizam alteraçóes agudas que interferem na função renal. ${ }^{(5,6)}$

Além disso, e frente à necessidade de melhor padronização dos critérios utilizados pelos diferentes estudos, grupo de especialistas da Acute Dialysis
Quality Initiative (ADQI) estabeleceram o critério RIFLE: risk (R: risco), injury (I: injúria), failure (F: falência), loss (L: perda mantida da função) e end-stage kidney disease (E: insuficiência renal terminal); e, posteriormente, especialistas do Acute Kidney Injury Network (AKIN) publicou nova classificação de LRA para adultos, considerada evolução do RIFLE. Por fim, as diretrizes do Kidney Disease Improving Global Outcomes (KDIGO), publicadas em 2012, incorporam os critérios RIFLE e AKIN, caracterizando a LRA pelo aumento igual ou superior a três décimos de miligramas por decilitro $(0,3$ $\mathrm{mg} / \mathrm{dL}$ ) da creatinina sérica em período de 48 horas, ou de uma vez e meia em relação ao nível basal conhecido ou preestabelecido ou que se presume ter ocorrido dentro dos últimos sete dias ou ainda fluxo urinário menor que meio mililitros por quilo por hora $(0,5 \mathrm{~mL} / \mathrm{kg} / \mathrm{h})$ por seis horas. ${ }^{(6,7)}$

Ao partir-se do pressuposto de que a LRA piora o prognóstico de pacientes em UTI e que as estratégias de prevenção e tratamento precoce asseguram melhor evolução do quadro clínico, ${ }^{(1-3)}$ é fundamental a atuação de equipe multiprofissional especializada para minimizar complicações e iniciar precocemente o tratamento adequado a cada caso, no cenário intensivo.

Ênfase deve ser atribuída à equipe de enfermagem, uma vez que se configura como os maiores provedores dos cuidados assistenciais especializados. Pode-se afirmar que o enfermeiro tem papel subtan- 
cial na prevenção da LRA em UTI, o qual, quando fundamentado em conhecimento qualificado, atua frente às diferentes complexidades e complicaçóes desencadeadas pelo tratamento ou pelas condiçóes intrínsecas do paciente clínico crítico, e através de condutas sistematizadas, contribui com a diminuição de intercorrências, bem como com a detecção precoce da deterioração da função renal. ${ }^{(8,9)} \mathrm{O}$ diagnóstico de enfermagem propicia atenção diferenciada, auxiliando no processo de tomada de decisão em relação às situaçóes para melhora da assistência multiprofissional, haja vista que o cuidado ao indivíduo com LRA deve ser prioridade a ser alcançada, para promoção da segurança deste. ${ }^{(10)}$

No entanto, apesar da complexidade e gravidade do tema, este ainda é pouco abordado na literatura de enfermagem e poucos estudos comparam pacientes acometidos de LRA com respectivos controles, para justificar os resultados encontrados. É nesta lacuna que se realizou a presente investigação, na tentativa de responder aos questionamentos: quais os fatores associados à LRA em pacientes clínicos críticos? Há diferença entre os fatores associados aos pacientes clínicos críticos quando comparados com grupo controle? A coexistência de fatores de risco é preditor de LRA em UTI? Qual a prevalência de LRA? Frente ao exposto, este estudo objetivou identificar prevalência e fatores associados à LRA em pacientes clínicos críticos, e compará-los com grupo controle; e analisar se a coexistência de fatores é preditor de risco para o desenvolvimento de LRA.

\section{Métodos}

Tratou-se de estudo tipo caso-controle, exploratório, retrospectivo e com abordagem quantitativa. Foi realizado em UTI adulto geral, de hospital privado do interior paulista, com capacidade para até 28 leitos para atendimento de pacientes provenientes dos atendimentos pré-hospitalares, unidade de pronto atendimento, centro cirúrgico, hemodinâmica, centros de diagnóstico e unidades de internação. No período do estudo, estavam ativos 20 leitos, distribuídos para assistência clínica e cirúrgica.
Amostra não probabilística foi constituída por pacientes que desenvolveram LRA e estiveram internados nesta unidade, durante os anos de 2014 e 2015.

Para os casos, foram considerados elegíveis pacientes com idade igual ou superior a 18 anos, de ambos os sexos que apresentaram aumento de 0,3 miligramas por decilitro $(\mathrm{mg} / \mathrm{dl})$ sobre o valor basal de creatinina sérica nas primeiras 48 horas de internação na UTI, conforme definição adotada na classificação KDIGO, pelo critério de creatinina.

Considerou-se como creatinina basal o último valor determinado pela medida laboratorial da instituição, anterior à internação na UTI, que antecedeu a 30 dias e náo ultrapassou seis meses da data de admissão na unidade. Para aqueles pacientes que não possuíam exame prévio de creatinina, considerou-se o primeiro valor colhido na admissão do paciente no hospital e, também, quando não disponível, o primeiro da internação na UTI. Foram excluídos os portadores de doença renal crônica documentada no prontuário e as reinternaçóes.

Adotou-se apenas o critério creatinina para estratificação dos pacientes, por considerar o fluxo urinário variável dinâmica e influenciada por inúmeros fatores clínicos e hemodinâmicos.

$\mathrm{E}$, como controles, foram incluídos pacientes que estiveram internados na UTI, no período do estudo, e não desenvolveram LRA. O pareamento dos controles foi realizado utilizando-se como critério a idade média $( \pm \mathrm{DP})$ dos pacientes casos. A amostra dos controles foi definida em função do número de casos identificados no período de estudo. Os pacientes casos que desenvolveram LRA compuseram o grupo "LRA", enquanto que os pacientes controles, o grupo "Sem LRA".

Os dados foram coletados de janeiro a novembro de 2016 pelo pesquisador, mediante consulta ao prontuário eletrônico dos pacientes, viabilizado por dois sistemas informatizados: Phostos e MedWiew. Através destes, e aplicando alguns filtros para pesquisa, obteve-se lista de internaçóes contendo o nome, número do prontuário e registro de atendimento.

A seguir, realizou-se o acesso ao sistema de resultados de exames laboratoriais, denominado Lhaudos, para identificação da disponibilidade de dados referentes à creatinina sérica. E, posterior checagem dos 
critérios de inclusão e exclusão, bem como constatação de LRA, conforme o critério KDIGO.

Para ambos os grupos, utilizou-se para coletar os dados um formulário composto de seis partes: dados de identificação do paciente (sexo, idade, cor, estado civil, dias de internação), variáveis da hospitalização (dias de internação, desdobramento da hospitalização, uso de ventilação mecânica, diagnóstico de entrada), antecedentes e/ou condiçôes clínicas (fatores de risco cardiovascular e outras comorbidades, fatores de risco nefrológicos), procedimentos realizados (vascular e contrastado), medicamentos nefrotóxicos/utilização de antibióticos e exames laboratoriais.

Como medicamentos nefrotóxicos, considerou-se a utilização de furosemida, anti-inflamatório não esteroidal ou hormonal, inibidores da enzima conversora de angiotensinogênio, quimioterápicos e antibióticos, classificados em: $\beta$-lactâmicos, quinolonas, glicopeptídeos, lipopeptídeos, polipeptídeos, oxazolidinonas, aminoglicosídeos, macrolídeos, lincosaminas, sulfonamidas, nitroimidazólicos, antivirais, antifúngicos. ${ }^{(11-16)}$

Com base nos critérios de inclusão, foram selecionados 205 pacientes que desenvolveram LRA no período do estudo e o mesmo número de controles.

Neste estudo, as variáveis quantitativas, como idade e tempo de seguimento na instituição, foram analisadas em termos de médias e desvios-padrão.

As demais variáveis, classificatórias, como sexo, cor, estado civil, diagnósticos de entrada, desfechos, fatores associados (cardiovasculares, nefrológicos, comorbidades e medicamentos) e valores laboratoriais, foram apresentadas em tabelas, contendo frequências absolutas (n) e relativas (\%).

A análise estatística ocorreu em duas etapas, utilizando-se do software SPSS 21. As variáveis que apresentaram significância estatística na análise univariada $(p<0,20)$ e relatadas pela literatura como potencial fator de risco para LRA foram utilizadas no ajuste do modelo de regressão logística múltipla. Os valores de $\mathrm{p}<0,05$ (intervalo de confiança IC 95\%) foram considerados estatisticamente significantes.

$\mathrm{Na}$ segunda etapa, foi realizado o teste de interaçôes duplas entre as exposiçôes incluídas no modelo múltiplo, e o modelo final foi composto somente com os principais efeitos de cada exposição e que, nesta etapa, sustentaram valor de associação para predição do aumento das chances frente à variável dependente, com base no conhecimento da relação com grupo de variáveis independentes consideradas estatisticamente significantes, gerando, assim, odds ratio da LRA entre pacientes clínicos críticos.

O estudo foi aprovado pelo Comitê de Ética da Faculdade de Medicina de Botucatu, conforme parecer $n^{\circ}$ 1.246.910 e CAE 48567315.2.0000.5411.

\section{Resultados}

Com base nos critérios de inclusão, foram selecionados 410 sujeitos que compuseram a amostra do estudo, sendo 205 em cada grupo. Na tabela 1 , estão apresentadas as características sociodemográficas dos participantes do estudo. Prevaleceram participantes do sexo feminino $210(51,2 \%)$, que tinham companheiro (a) 221 (53,9\%) e eram caucasianos 354 (86,3\%). No que concerne aos diagnósticos de entrada, sobressaíram os respiratórios $118(28,8 \%)$ e cardiovasculares 106 (25,9\%), em ambos os grupos, com diferença estatisticamente significante ( $\mathrm{p}=0,0178$ e $\mathrm{p}=0,0008$, respectivamente). Verificou-se, também, que o percentual de participantes que utilizaram ventilação mecânica foi maior no grupo LRA 127 (61,9\%), e que também tiveram maior tempo médio de internação 9,8 dias (DP $\pm 10,2$ dias), ambos estatisticamente significantes $(\mathrm{p}<0,0001)$. Praticamente, a metade 96 (46,8\%) tinha como classificação estágio I de acometimento renal e o óbito $136(66,4 \%)$ foi o desfecho significante neste grupo $(\mathrm{p}<0,0001)$. A prevalência de LRA foi estimada em $7,5 \%$.

$\mathrm{Na}$ tabela 2, estão apresentados os dados referentes à análise de regressão logística das variáveis estatisticamente significantes para o desenvolvimento de LRA entre os pacientes do grupo LRA. Verificouse que dentre as doenças de base, a hipertensão arterial $(p=0,0349 ; O R=1,9615 ; \mathrm{IC}=1,0491-3,6645)$; e insuficiência cardíaca $(\mathrm{p}=0,0032 ; \mathrm{OR}=5,3123$; $\mathrm{IC}=1,7521-16,1051)$ despontaram como fatores de risco para LRA, aumentando em quase duas e mais de cinco vezes as chances para o desenvolvimento 
Tabela 1. Variáveis sociodemográficas, clínicas e desdobramentos da hospitalização dos participantes do estudo

\begin{tabular}{|c|c|c|c|c|}
\hline Variáveis & $\begin{array}{l}\text { LRA } \\
\text { n(\%) }\end{array}$ & $\begin{array}{c}\text { Sem LRA } \\
\mathrm{n}(\%)\end{array}$ & $\begin{array}{l}\text { Total } \\
\mathrm{n}(\%)\end{array}$ & $p$-value \\
\hline Idade $(\operatorname{anos})^{*}$ & $74,6(15,5)$ & $72,4(13,8)$ & $73,5(14,7)$ & 0,1784 \\
\hline Dias de internação* & $9,8(10,2)$ & $4,6(6,1)$ & $7,2(8,7)$ & $<0,0001$ \\
\hline \multicolumn{5}{|l|}{ Sexo } \\
\hline Feminino & $102(49,8)$ & $108(52,7)$ & $210(51,2)$ & \multirow[t]{2}{*}{0,5538} \\
\hline Masculino & $103(50,2)$ & $97(47,3)$ & $200(48,8)$ & \\
\hline \multicolumn{5}{|l|}{ Estado civil } \\
\hline Com companheiro (a) & $107(52,2)$ & $114(55,6)$ & $221(53,9)$ & \multirow[t]{2}{*}{0,4885} \\
\hline Sem companheiro (a) & $98(47,8)$ & $91(44,4)$ & $189(46,1)$ & \\
\hline \multicolumn{5}{|l|}{ Raça } \\
\hline Caucasiana & $182(88,8)$ & $172(83,9)$ & $354(86,3)$ & \multirow[t]{2}{*}{0,1509} \\
\hline Não caucasiana & $23(11,2)$ & $33(16,1)$ & $56(13,7)$ & \\
\hline \multicolumn{5}{|l|}{ Diagnósticos de entrada } \\
\hline Cardiovascular & & & & \\
\hline Sim & $38(18,5)$ & $68(33,2)$ & $106(25,9)$ & \multirow[t]{2}{*}{0,0008} \\
\hline Não & $167(81,5)$ & $137(66,8)$ & $304(74,1)$ & \\
\hline \multicolumn{5}{|l|}{ Gastrointestinal } \\
\hline $\operatorname{Sim}$ & $32(15,6)$ & $23(11,2)$ & $55(13,4)$ & \multirow[t]{2}{*}{0,1951} \\
\hline Não & $173(84,4)$ & $182(88,8)$ & $355(86,6)$ & \\
\hline \multicolumn{5}{|l|}{ Neurológico } \\
\hline Sim & $30(14,6)$ & $40(19,5)$ & $70(17,1)$ & \multirow[t]{2}{*}{0,1918} \\
\hline Não & $175(85,4)$ & $165(80,5)$ & $340(82,9)$ & \\
\hline \multicolumn{5}{|l|}{ Respiratório } \\
\hline Sim & $70(34,1)$ & $48(23,4)$ & $118(28,8)$ & \multirow[t]{2}{*}{0,0178} \\
\hline Não & $135(65,9)$ & $157(76,6)$ & $292(71,2)$ & \\
\hline \multicolumn{5}{|l|}{ Trauma / Ortopedia } \\
\hline Sim & $4(2,0)$ & $6(2,9)$ & $10(2,4)$ & \multirow[t]{2}{*}{0,5250} \\
\hline Não & $201(98,0)$ & $199(97,1)$ & 400(97,6) & \\
\hline \multicolumn{5}{|l|}{ Urinário } \\
\hline Sim & $22(10,7)$ & $12(5,9)$ & $34(8,3)$ & \multirow[t]{2}{*}{0,0737} \\
\hline Não & $183(89,3)$ & $193(94,1)$ & $376(91,7)$ & \\
\hline \multicolumn{5}{|l|}{ Outros } \\
\hline Sim & $9(4,4)$ & $8(3,9)$ & $17(4,2)$ & \multirow[t]{2}{*}{0,8367} \\
\hline Não & $196(95,6)$ & $197(96,1)$ & $393(95,8)$ & \\
\hline \multicolumn{5}{|l|}{ Uso ventilação mecânica } \\
\hline Sim & $127(61,9)$ & $25(12,2)$ & $152(37,1)$ & \multirow[t]{2}{*}{$<0,0001$} \\
\hline Não & $78(38,1)$ & $180(87,8)$ & $258(62,9)$ & \\
\hline \multicolumn{5}{|l|}{ Classificação KDIGO } \\
\hline । & $96(46,8)$ & - & - & - \\
\hline$\|$ & $71(34,7)$ & & & \\
\hline |II & $38(18,5)$ & - & - & - \\
\hline \multicolumn{5}{|l|}{$\begin{array}{l}\text { Desdobramentos } \\
\text { da hospitalização }\end{array}$} \\
\hline Óbito & $136(66,4)$ & $29(14,2)$ & $165(40,3)$ & $<0,0001$ \\
\hline
\end{tabular}

* Média (Desvio Padrão); KDIGO - Kidney Disease Improving Global Outcomes

da doença, respectivamente. Condições hemodinâmicas associadas à hipovolemia aumentaram as chances para LRA em cinco vezes $(p=0,0060$; $\mathrm{OR}=5,6071 ; \mathrm{IC}=1,6382-19,1854)$. Quanto aos medicamentos com potencial nefrotóxico utilizados pelos participantes, observou-se que o uso noradrenalina $(\mathrm{p}<0,0001 ; \mathrm{OR}=9,4913 ; \mathrm{IC}=4,4824$ 20,0981); dopamina ( $\mathrm{p}=0,0009 ; \quad \mathrm{OR}=5,2612$; $\mathrm{IC}=1,6701-7,4242) ; \quad$ dobutamina $\quad(\mathrm{p}=0,0131$; $\mathrm{OR}=5,2612 ; \mathrm{IC}=1,4172-19,5323)$ e antibióticos simultâneos（ $\mathrm{p}<0,0001 ; \mathrm{OR}=3,7881 ; \mathrm{IC}=2,0253$ $7,0884)$ aumentaram as chances dos participantes desenvolverem LRA em nove, três, cinco e quatro vezes, respectivamente.

Tabela 2. Regressão logística dos fatores de risco para LRA em pacientes clínicos críticos

\begin{tabular}{lcccc}
\hline Variáveis & $\begin{array}{l}\text { Odds } \\
\text { Ratio }\end{array}$ & \multicolumn{2}{c}{ IC (95\%) } & p-value \\
\hline Hipertensão arterial & 1,9615 & 1,0491 & 3,6645 & 0,0349 \\
Hipovolemia & 5,6071 & 1,6382 & 19,1854 & 0,0060 \\
Insuficiência cardíaca & 5,3123 & 1,7521 & 16,1051 & 0,0032 \\
Noradrenalina & 9,4913 & 4,4824 & 20,0981 & $<0,0001$ \\
Dopamina & 3,5212 & 1,6701 & 7,4242 & 0,0009 \\
Dobutamina & 5,2612 & 1,4172 & 19,5323 & 0,0131 \\
Dois ou mais antibiótico simultâneos & 3,7881 & 2,0253 & 7,0884 & $<0,0001$ \\
\hline
\end{tabular}

Na tabela 3, está descrita a coexistência de fatores associados com o desenvolvimento de LRA. A maioria dos participantes do grupo LRA (59,5\%) apresentou mais de três fatores coexistentes durante a hospitalização na UTI no período investigado, o que contribuiu para aumentar em cinco vezes a chance dos participantes desenvolveram LRA ( $\mathrm{p}<0,0001 ; \mathrm{OR}=5,0074 ; \mathrm{IC}=2,5601-9,7936)$.

Tabela 3. Fatores de risco coexistentes dos participantes do estudo

\begin{tabular}{lccccccc}
\hline Fatores & $\begin{array}{c}\text { LRA } \\
\mathbf{n}(\%)\end{array}$ & $\begin{array}{c}\text { Sem LRA } \\
\mathrm{n}(\%)\end{array}$ & $\begin{array}{c}\text { Total } \\
\mathbf{n}(\%)\end{array}$ & $\begin{array}{c}\text { Odds } \\
\text { Ratio }\end{array}$ & IC (95\%) & p-value \\
\hline$>3$ & $122(59,5)$ & $21(10,2)$ & $143(34,9)$ & 5,0074 & 2,5601 & 9,7936 & $<0,0001$ \\
\hline IC - Intervalo de confiança
\end{tabular}

Discussão

Os resultados deste estudo possibilitaram identificar os principais fatores associados à LRA, além de contribuírem para reflexão acerca da importância da assistência prestada pela equipe multiprofissional, especialmente de enfermagem, na prevenção e no controle de agravos a pacientes internados em UTI.

Além disso, pode contribuir para subsidiar a prática assistencial, por meio da elaboraçáo de protocolos assistenciais para detecção precoce e acompanhamento da evolução da LRA em pacientes clínicos.

Destaca-se como limitação do estudo o fato de ter sido realizado em único hospital privado. Observaram-se, ainda, a carência de registros em prontuário eletrônico, a ausência de índice de gravidade dos pacientes e a não utilização de escalas padronizadas na literatura, para identificação de LRA. 
Destacaram-se participantes com faixa etária elevada, caucasianos e que tinham companheiro. Dados corroborados com outros estudos cuja injúria renal foi maior entre pacientes com idade média acima dos $70 \operatorname{anos}^{(4)}$ e com a mesma característica racial. ${ }^{(5)}$

No que se refere aos desfechos, o tempo de internação e o óbito foram eventos significativos. $\mathrm{O}$ prolongamento da permanência na UTI pode afetar negativamente o estado de saúde, aumentando o risco de complicaçóes e a mortalidade, como demonstrado em estudos recentes. ${ }^{(2,5,8,9)}$ Além do que, notase associação entre progressão da LRA, aumento da deterioração clínica e escore de gravidade, ${ }^{(2)}$ sendo esta condição diretamente relacionada com a piora do prognóstico e óbito. Além disso, cabe considerar que quando o acometimento renal passa a ser estratificado, como na proposta KDIGO, torna-se mais evidente a complexidade dos pacientes em detrimento dos estágios de estadiamento da nefropatia vivenciada. ${ }^{(7,8)}$

A elevada mortalidade identificada no presente estudo elucida a necessidade de um melhor atendimento a essa população, bem como identificação dos grupos de maior risco para monitorização, detecção precoce e adoção de medidas preventivas.

Ademais, o tempo de internação na UTI, a partir de cinco dias, aliado à necessidade de ventilação mecânica e cirurgia de emergência, incrementa o risco para desenvolvimento de doenças graves, incluindo deterioração da função renal ${ }^{(17)}$ Neste estudo, a média de permanência dos pacientes com LRA foi de aproximadamente dez dias.

No tocante aos diagnósticos de entrada, destacaram-se as doenças respiratórias como principal motivo associado ao desenvolvimento de LRA entre os pacientes investigados. Fato que provavelmente tenha contribuído para dependência ventilatória e demais complicaçóes advindas com o prolongamento da internação. Estudo de coorte prospectiva, realizado com 27 pacientes em hospital público, mostrou que as terapias ventilatórias estão associadas com alteraçôes no débito cardíaco e no ritmo da filtração glomerular, principalmente quando empregada pressão positiva expiratória final mais elevada. ${ }^{(17)}$
Pelos dados analisados, praticamente a metade dos investigados com LRA tinha estágio I de KDIGO de acometimento renal, corroborando com dados de estudo retrospectivo realizado com 157 pacientes críticos $^{(18)}$ e coorte de 2,719 paciente. ${ }^{(19)}$ Outras pesquisas também demostram variação entre as classificaçóes, sobretudo quando analisado o grau máximo da disfunção, como evidenciado em estudo abrangendo perfis clínicos e relação com processos infecciosos graves que constatou percentuais elevados de KDIGO II e III. ${ }^{(19)}$ Enfatiza-se que na instituição lócus da pesquisa a utilização desta escala não faz parte da rotina institucional. No entanto, para assegurar o rigor metodológico da pesquisa, este levantamento caso a caso foi realizado pelos pesquisadores.

Para responder ao segundo objetivo deste estudo, realizou-se a regressão logística dos pacientes que desenvolveram LRA, bem como do grupo controle sem LRA, com algumas variáveis explanatórias. Dentre os fatores de risco cardiovascular, destacaram-se como estatisticamente significantes a hipertensão arterial e a insuficiência cardíaca congestiva. Dado confirmado em pesquisa realizada com 37 pacientes gravemente enfermos, cujos fatores foram descritos como coadjuvantes da disfunção renal. ${ }^{(20)}$ Aponta-se que a hipertensão arterial e o diabetes, são considerados os principais fatores associados ao desenvolvimento de LRA. ${ }^{(1,2,10,18)}$ Ademais, a ocorrência de alteraçóes hemodinâmicas, associadas com hipovolemia, e utilização de agentes nefrotóxicos também repercutiram significativamente para LRA nesta investigação.

Neste estudo, a antibioticoterapia quadriplicou as chances para acometimento renal, sobretudo entre os que utilizaram tais medicamentos de forma simultânea, ou seja, mais de uma classe de antibióticos concomitantemente. Dentre as principais classes de risco para o desenvolvimento de LRA, os aminoglicosídeos, ${ }^{(13)}$ os glicopeptídeos e as polimixinas $^{(6,20,21)}$ são consagrados na literatura.

Atuando como coadjuvante do potencial nefrotóxico, as drogas vasoativas também foram associadas com o desenvolvimento de LRA entre os participantes deste estudo. Estudo retrospectivo, com análise de prontuário de 74 pacientes, verificou 
prevalência do uso destas drogas dentre os participantes com acometimento renal e risco para óbito, cuja associação com maior gravidade e mortalidade foi significativa. Além disso, constitui-se como uma das possíveis causas de lesão renal, principalmente quando utilizada em concomitância com outros agentes nefrotóxicos. ${ }^{(5)}$

Destaca-se que a nefrotoxicidade é causada em decorrência do efeito vasoconstritor que indiretamente gera isquemia, com consequente diminuição do fluxo sanguíneo renal, com necessidade de cautela quanto aos efeitos deletérios, seja pelas dosagens ou tempo de uso prolongado. ${ }^{(5)}$ Tal condição requer vigilância constante, sobretudo quanto ao monitoramento da função renal, através de dosagens de ureia, creatinina e débito urinário. ${ }^{(7,8)}$

Neste estudo, apesar dos fatores de risco estatisticamente significantes para o desenvolvimento de LRA, a prevalência foi relativamente baixa quando comparada a outras pesquisas, nas quais a disfunção renal variou entre 23 e $53 \% .^{(2,10,18,19,21)}$ Apenas em um estudo retrospectivo recente este percentual assemelhou-se ao da presente investigação (8\%). ${ }^{(13)}$ Cabe considerar que a ausência de padronização do diagnóstico da LRA dentre as publicaçóes consultadas repercutiu diretamente nas prevalências identificadas, representando portanto um fator limitante quanto à sua classificação.

$\mathrm{Na}$ presente investigação, praticamente $60 \%$ dos participantes do grupo LRA apresentaram mais de três fatores coexistentes, o que contribuiu para aumentar o risco de disfunção renal em mais de cinco vezes. Por outro lado, a coexistência dos fatores de risco é pouco explorada na literatura, e quando referenciada, apresenta dados relacionados à combinação de alguns deles, como em estudo retrospectivo $^{(13)}$ acerca do uso de vancomicina e drogas nefrotóxicas, considerando ainda a combinação destes fatores com o uso combinado de outros antibióticos e contraste radiológico.

Reitera-se que quando coexistentes, os fatores associados geram maior impacto para o acometimento renal, ${ }^{(2,4,5)}$ e que a LRA resulta da sinergia de fatores intrínsecos e extrínsecos, constituindo condição que repercute no prognóstico de pacientes clínicos críticos. ${ }^{(8,12,19)}$

\section{Conclusão}

A LRA em pacientes clínicos críticos é um evento multifatorial, que ocorre notadamente em pacientes com idade avançada, com maior tempo de internação, e predispóe ao óbito. Associou-se a doenças de base, complicaçóes decorrentes da gravidade dos participantes e utilização de medicamentos nefrotóxicos. A prevalência foi baixa em relação à literatura. Ademais, o estudo também mostrou que a coexistência de mais de três fatores de risco contribuiu para o desenvolvimento de LRA.

\section{Colaborações}

Benichel CR declara que contribuiu com concepção do projeto, análise e interpretação dos dados, redação do artigo e revisão crítica relevante do conteúdo intelectual e aprovação final da versão a ser publicada. Meneguin S declara que contribuiu com concepção do projeto, análise e interpretação dos dados, redação do artigo, revisão crítica relevante do conteúdo intelectual e aprovação final da versão a ser publicada.

\section{Referências}

1. Sociedade Brasileira de Nefrologia. Comitê de Insuficiência Renal Aguda. Diretrizes da Sociedade Brasileira de Nefrologia: Insuficiência renal aguda. [Internet]. São Paulo: Sociedade Brasileira de Nefrologia; 2007 [citado 2018 Out 17]. Disponível em: http://sbn.org.br/app/ uploads/Diretrizes_Insuficiencia_Renal_Aguda.pdf.

2. Peres $L A$, Wandeur $V$, Matsuo T. Predictors of acute kidney injury and mortality in an Intensive Care Unit. J Bras Nefrol. 2015;37(1):38-46.

3. Horkan CM, Purtle SW, Mendu ML, Moromizato T, Gibbons FK, Christopher KB. The association of acute kidney injury in the critically ill and postdischarge outcomes: a cohort study. Crit Care Med. 2015 ;43(2):354-64.

4. Kane-Gill SL, Sileanu FE, Murugan R, Trietley GS, Handler SM, Kellum JA. Risk factors for acute kidney injury in older adults with critical illness: a retrospective cohort study. Am J Kidney Dis. 2015;65(6):8609

5. Luft J, Boes AA, Lazzari DD, Nascimento ER, Busana JA, Canever BP. Kidney injury at an intensive care service: clinical characteristics and outcomes. Cogitare Enferm. 2016;21(2):1-9.

6. Vasco CF, Watanabe M, Fonseca CD, Vattimo MF. Sepsis-induced acute kidney injury: kidney protection effects by antioxidants. Rev Bras Enferm. 2018;71(4):2032-8 
7. Kidney Disease: Improving Global Outcomes (KDIGO) Acute Kidney Injury Work Group. KDIGO clinical practice guideline for acute kidney injury. Kidney Int Suppl. 2012;2:1-138.

8. Silva CM, Silva DA, Silva GG, Maia LF, Oliveira TS. Acute renal failure: main causes and the ICU nursing intervention. Rev Recien. 2016;6(16):48-56. Available from: https://www.recien.com.br/index. php/Recien/article/view/134/204.

9. Nascimento RA, Assunção MS, Silva Junior JM, Amendola CP, Carvalho $T M$, Lima EQ, et al. Nurses' knowledge to identify early acute kidney injury. Rev Esc Enferm. 2016;50(3):399-404.

10. Rigonatto MC, Magro MC. Risk for acute kidney injury in primary health care. Rev Bras Enferm. 2018;71(1):25-30.

11. Pontes LB, Antunes YP, Bugano DD, Karnakis T, Giglio AD, Kaliks RA. Prevalence of renal insufficiency in elderly cancer patients in a tertiary cancer center. einstein (São Paulo). 2014;12(3):300-3.

12. Lucas GN, Leitão AC, Alencar RL, Xavier RM, Daher EF, Silva Junior GB. Pathophysiological aspects of nephropathy caused by non-steroidal anti-inflammatory drugs. J Bras Nefrol. 2018. Available

13. Hanrahan TP, Kotapati C, Roberts MJ, Rowland J, Lipman J, Roberts $\mathrm{JA}$, et al. Factors associated with vancomycin nephrotoxicity in the critically ill. Anaesth Intensive Care Med. 2015;43:594-99.

14. Guimarães Y, Rocha MS, Moura CA, Moura CG, Cruz CM. Acute kidney injury in patients using angiotensin-converting enzyme inhibitorsto treat decompensated heart failure. Rev Soc Bras Clin Med. 2014;12(4):1-7.
15. Shahrbaf FG, Assadi F. Drug-induced renal disorders. J Renal Inj Prev. 2015;4(3):57-60.

16. Agência Nacional de Vigilância Sanitária (ANVISA). Antimicrobianos: bases teóricas e uso clínico [Internet]. Brasília (DF): ANVISA; 2007 [citado 2019 Jan 15]. Disponível em: http://www.anvisa.gov.br/ servicosaude/controle/rede_rm/cursos/rm_controle/opas_web/ modulo1/conceitos.htm.

17. Santos LL, Magro MC. Mechanical ventilation and acute kidney injury in patients in the intensive care unit. Acta Paul Enferm. 2015; 28(2):14651.

18. Moura EL, Amorim FR, Huang W, Maia MO. Contrast-induced acute kidney injury: the importance of diagnostic criteria for establishing prevalence and prognosis in the intensive care unit. Rev Bras Ter Intensiva. 2017;29(3):303-9.

19. Vaara ST, Pettilä V, Kaukonen K, Bendel S, Korhonen A, Bellomo $R$, et al. The attributable mortality of acute kidney injury: a sequentially matched analysis. Crit Care Med. 2014;42(4):87885.

20. Schmidt L, Wiese LP, Pereira EM, Possamai KS, Santos M, Fernandes FM. Acute kidney injury in critical patients: profile clinical and relationship with serious processes infectious. Rev Bras Farm Hosp Serv Saúde. 2016;7(3):19-24.

21. Bahlis LF, Diogo LP, Lemons D, Klaus D. Risk factors for acute kidney injury in patients treated with polymyxin B at a Tertiary Care Medical Center. J Bras Nefrol. 2015;37(4):446-50. 